\title{
Extended critical situations: the physical singularity of life phenomena ${ }^{1}$
}

\author{
Francis Bailly \\ Physique, CNRS, Meudon
}

\author{
Giuseppe Longo \\ LIENS, CNRS - ENS et CREA, Paris \\ http://www.di.ens.fr/users/longo
}

\begin{abstract}
In this paper we propose to consider living systems as "coherent critical structures", though extended in space and time, their unity being ensured through global causal relations between levels of organization (integration/regulation). This may be seen as a further contribution to the large amount of work already done on the theme of selforganized criticality. More precisely, our main physical paradigm is provided by the analysis of "phase transitions", as this peculiar form of critical state presents interesting aspect of emergence: the formation of extended correlation lengths and coherence structures, the divergence of some observables with respect to the control parameter(s), etc.... However, the "coherent critical structures" which are the main focus of our work cannot be reduced to existing physical approaches, since phase transitions, in physics, are treated as "singular events", corresponding to a specific well-defined value of the control parameter. Whereas our claim is that in the case of living systems, these coherent critical structures are "extended" and organized in such a way that they persist in space and time. The relation of this concept to the theory of autopoiesis, as well as to various forms of teleonomy, often present in biological analyses, will be also discussed.
\end{abstract}

Keywords: criticality, extended phase transition, coherence structure, autopoiesis.

\section{Classification:}

Primary: 1.000 Theoretical Biology

Secondary: 2.100 Philosophy of Biology.

\section{Methodological preliminaries.}

Mathematics has a normative role for physics: it organizes reality, particularly since the advent of infinitesimal calculus (the role of differential equations, for instance) and, later on, through the geometrization of physics. It ought to play a similar role, if possible, for other scientific disciplines. The common idea according to which the same mathematical tools, so successful with regard to physics, can play a similar role for biology is often based on a confusion between the dependency of biological phenomena upon physical phenomena, and their reducibility to such physical phenomena. The fact that the phenomena pertaining to living matter are dependent upon physical structure is a basic presupposition for any monist. However, reduction to physics is a theoretical operation: living matter is reduced to a conceptual organization which is given and specific to inertia. The first aspect is intimately

\footnotetext{
${ }^{1}$ In Journal of Biological Systems (JBS), vo. 16, 2, 309 - 336, June 2008. A preliminary French version of this paper appeared, as invited lecture, in the proceedings of "Détermination et complexité", (P. Bourgine, ed.) Colloque de Cerisy, Juin 2004 (La Découverte, Paris, 2007) and as chapter 6 in [Bailly, Longo, 2006].
} 
related to the presupposed foundations of modern science; the second aspect must be implemented and this is generally the case when reference is made to the existing physical theories, which may prove inadequate to achieve this. In this text, and in the view of unifying different facts, we will attempt to better clarify some organizing concepts of a number of biological phenomena. This is, once again, a rather modest attempt at "unification through concepts" - an attempt which should always precede the process of mathematization - by returning to an idea already formulated elsewhere, that of the "criticality" of the living; we "extend" this concept in a way beyond the reach of current physical theories. In our opinion, this concept may prove to be an interpretational key by which to grasp biological phenomenality, in a number of situations. We insist here on the importance of a preliminary construction of a conceptual frame; it is a hindrance rather than a help to adequate mathematical formulation if lack of sufficient time precipitates the performance of calculations before understanding and conceptualizing. As a matter of fact, mathematics itself is the result of a progressive conceptualization, where crucial notions and structures result from a difficult stabilization of informal conceptual practices. In this vein, we are attempting here to unify some biological phenomena and structures, by using relatively technical concepts but no explicit mathematics; we are only suggesting by this a possible mathematization. In this trans-disciplinary attempt, we are thus drawing on our experience of "theorization" in the field of mathematics and physics by orienting it towards biology. Our experience differs indeed from the practice of biology, but nevertheless makes it possible to distinguish new approaches which we hope to develop on the basis of a sustained and rigorous dialogue with practitioners in this field.

\section{Introduction: singularities and criticality in physics.}

\subsection{From gas to crystal.}

A crystal and a gas are opposite to one another, in what concerns the spatial ordering of their components. In physics, the analysis of the passing from one to another, of the phase transition, involves specific concepts: those of critical exponents, of divergence or of the discontinuity of certain quantities related to the system (susceptibilities), of increases in fluctuations, of divergence of correlation lengths, etc.

These notions are associated to the passing through a critical state, which radically changes the relevant properties and parameters used to describe the object studied. Particularly, as we have just mentioned, the phase transitions change the correlation lengths which, in certain cases, may be interpreted as a change in the scope of (possibly causal) relationships or of the "coherence structure" between the elements of the studied physical structure. During the process of change of state, the global structure is completely involved in the behavior of the elements: the local situation depends upon (is correlated to) the global situation (see [Barenblatt, 1996], [Jensen, 1998]). Mathematically, this may be expressed by the fact that this correlation length formally tends towards infinity, for example in the case with first order transitions, such as para-/ferro-magnetic transition; physically, this means that the determination is global and not local. However, the transition sometimes occurs prior to complete divergence, when the length in question remains finite, like in the case with first order transitions, such as liquid to solid transitions, at a finite length. In any case, 
the most precise treatment usually recourses to the theory of the renormalization of measurements and of the parameters at transition point ${ }^{2}$.

As a matter of fact, in physics, a critical state is the most generally reducible to the critical point; renormalization, which enables to account for the passing from the local to the global, finds itself confined to the value of the parameter for which the transition occurred, which finally makes this renormalization implicitly dependent upon a single value for this parameter (putting aside here the dependency in function of the dimension of the space of embedding or that of the order parameter - in the examples mentioned, the magnetic momentum and the density).

To sum up, in physics, a critical state may be related to a change of phase and to the apparition of critical behaviors of some magnitudes of the system's states - magnetization, density, for example - or of some of its particular characteristics - such as correlation length. It is likely to appear at equilibrium (null fluxes) or far from equilibrium (non-null fluxes). If, in the first case, the mathematico-physical processing is rather well-understood and thermodynamics is used for the bridge between microscopic and macroscopic description; on the other hand, in the second case, far from equilibrium, we are far from having at our disposal theories as satisfactory, from the moment.

It is difficult to gather under a sole characterization the phenomena of the critical type, but a common signature is provided by the divergence tendencies of lengths and times of correlation as well as by a loss of analycity for functions of the system's state (such as free energy), manifested by the apparition of non-integral critical exponents, occurring in the phase transitions. Another common mathematical aspect is found in the fact that the set of critical points, which are located in the space of control parameters, is generally of null measure. It possibly is a discrete set of points, as observed above, relatively to the evolution the parameter(s) (the temperature, for instance, see also §.2.2.B). We can notice that the critical situation, in the case of the null flux, appears to be atemporal, whereas, far from equilibrium and because of fluxes, also when the system remains in a stationary state, there exists a natural time scale.

\subsection{From the local to the global.}

Let's return for a moment to certain characteristics which we wish to highlight and which will enable us to better apprehend the relationships between "local" and "global" as they continue to impose themselves upon the sphere of living phenomena from the moment that relationships of whole to parts are involved, via the processes of regulation and integration, among others. Thus, as we have just seen, during critical transitions (change of phases), several characteristics manifest the passage from the local to the global in relation to the relevance of the described objects and of their interaction:

- the divergence of the correlation length, which is a global statistical property, different but based also on local interactions - e.g. spin coupling;

- the apparition of an order, possibly measured by an order parameter which becomes non-null, a symmetry breaking;

\footnotetext{
${ }^{2}$ Renormalization describes a change in measurement and of object, obtained by integrating the new classes of interaction due to transition. Its properties stem from the fact that at critical transition, the passing to the infinite limit of the correlation length produces an invariance of scale for the system and a fixed point from its dynamic (see [Binney et al., 1992], [Delamotte, 2004] for an introduction).
} 
- the apparition of critical exponents associated to discontinuities or to divergences (susceptibilities);

- the sudden non-analycity of free energy, from the thermodynamic point of view, ....

In the case of the critical transition from liquid to crystal, for instance, an order or network appears, that of the crystalline structure, and density undergoes a discontinuity. Another classical example is the transition from the paramagnetic to the ferromagnetic in a spin system: when we approach the critical temperature, Tc (Curie point) the global magnetic moment, which serves here as order parameter, becomes non-null. Phenomenologically, it amounts to considering that the entropic component of free energy, dominant at high temperatures because of the thermal disorder thus generated, becomes secondary before the specifically energetic component due to the interaction between spins, of which the alignment diminishes this energy, all the while being at the origin of the apparition of the global magnetic moment. At the same time, since the effect of a perturbation from the $r$ distance is generally in $\exp (-\mathrm{r} / \mathrm{L})$, where $\mathrm{L}$ is the length of correlation, then the correlations acquire a scope which increases until involving the system's total volume. In the case under discussion, this correlation length L diverges as $(\mathrm{T} / \mathrm{Tc}-1)^{-v}$, where $\mathrm{Tc}$ is the critical temperature of transition and $v$ a critical exponent, often equal to $1 / 2$. Likewise, magnetic susceptibility diverges with its own critical exponent. We may comment this by saying that at the critical point, a finite cause induces an infinite effect, or that an infinitesimal produces a finite effect.

As we have mentioned earlier, these critical exponents themselves may be calculated by means of the technique of the "renormalization group" ([Delamotte, 2004]). This technique is made possible because of the infinite length of correlation which makes the system scale invariant at the critical point. It stems from these characteristics that the "relevant object", both for observation and for the theory, changes at the critical point: from local (the individual disordered spins, say, correlated only to the closest) the concerned scale becomes global (the magnetic moment of the system taken as a whole, because of the global scope of the correlations).

With these simple examples, we have seen at work the notions of critical state, of phase transition, of correlation length (see [Bak et al., 1988; Kauffman, 1993; Jensen, 1998] and their numerous examples and references ; more will be said below). The scale of observation thus matters in a crucial way with regard to the greatest possible distance of direct causal interactions between the elements of a system.

To conclude, the passing from the local to the global, in physics, necessitates the overstepping of a critical state ${ }^{3}$; this is understood as a mathematical divergence from the length of interaction, therefore as a point where infinitesimal variations create finite changes (or finite variations leading to mathematically finite changes).

From a conceptual point of view, because of the essentially global determination of a biological object with regard to its local components, we will stress the connection with these critical situations, but while considering them here as spatio-temporally extended, and by considering its boundaries (of physicochemical states) as co-constitutive of the biological

\footnotetext{
${ }^{3}$ We are speaking here of a passage which induces qualitative modifications (the critical transition "produces" a new physical object, like a magnet) rather than only a statistical processing at equilibrium (or even far from equilibrium) of a system with many degrees of freedom. This is the case for a gas within which one can nevertheless define "global" magnitudes by means of averages (temperature, pressure, entropy, etc.).
} 
entity. From this point of view, the existence and the maintenance of living organisms would then be assimilable to the existence and to the maintaining of a situation (or zone) of extended criticality. From the standpoint of complexity, and as we have already evoked previously, the result would be the situation where living matter, regardless of the level at which it is considered, would present an infinite complexity with regard to the physicochemical. Furthermore, living matter organizes itself into a hierarchy of these levels, according to several orders of infinity, which can be considered as successive processes of "conceptual renormalizations". In the section 2, we will attempt to develop this point of view.

\subsection{Phase transitions in self-organized criticality and "order for free".}

The role of critical transitions for the analysis of life phenomena has already been highlighted by other authors. The idea at the center of the approaches originating from the 1988 article by Bak, Tang and Wiesenfeld is that physics proposes several examples of construction of self-organization close to critical states and far from equilibrium, an idea that was already central to [Nicolis, Prigogine, 1977]. Another view regarding self-organized criticality sees it as emerging from chaos: "order for free" to use the terminology of [Kauffman, 1995] (see also [Solé, Goodwin, 2000]). In short, to use the point of view exposed in [Kauffman, 1995], the simple interaction between elements that are not organized a priori can be at the origin of the formation of organized structures. It is the network correlating simple elements which produces the emergence (a keyword in these approaches) of complex structures or phenomena. And this, by reaching a critical point: the early and paradigmatic case, analyzed in [Bak et al., 1988], is provided by the sand piles (dropping sand on a same point until the formation of a pile and then of avalanches).

The analyses which we will develop here share with these approaches, especially the one proposed by Kauffman, the role attributed to the emergence of coherent structures during "phase transitions", as a passage from one state, or process, to another. "Life may exist near a phase transition", as Kauffman [Kauffman, 1995] explicitly proposes: it appears to be located between order and chaos, at the "edge of chaos", because too much order, or equilibrium, corresponds to death, whereas chaos is the opposite of organization (and living phenomena is very organized). In the cases studied, the notion of "edge of chaos", a passing point between order and disorder, has a very specific physico-mathematical significance and always refers to a mathematical point in the space of the control parameters (or most often, in the space of the control parameter).

What is particularly important in these transitions, which are critical in the mentioned mathematical sense, is that the global structure, which constitutes itself (emerges) from the transition, is completely involved in the local activities and vice-versa: the magnet which forms at the critical temperature, at the Curie point, correlates the local spins and the global orientation. In short, the local situation depends on the global one, by a "coherent structure" emerging at the critical parameter. Mathematically, this is expressed by the fact that the correlation length formally tends towards infinity or that infinitesimal variations of the control parameter induce finite changes in the observables; physically, this means that the determination is now global, not local.

The idea of using the physics of criticality, with its "formation of coherent structures" to analyze the emergence of the structural stability of living phenomena, is also at the center of our analyses; because over the last twenty years, these physics of criticality have been developing an appealing theory of the emergence of organized forms in the presence of 
critical transitions. These structures "constitute themselves all alone" ("order for free"): hence, criticality self-organizes.

Here are a few characteristics of this self-organization, as seen by the authors we have mentioned:

- the existence of many stable states;

- the passing from the one to the other of these states may depend on fluctuations (infinitesimal ones);

- the existence of bifurcations, which present themselves at very specific values of the control parameters (critical values); by exceeding these exact values, and only in these cases, coherent structures appear.

In the paradigmatic case of the formation of the heap of sand, when a certain critical angle, a mathematical singularity, is reached by the addition of sand grains, avalanches will form, according to a very specific mathematical structuration. One of the limits of this example, at the center of the analyses by Per Bak, is that self-organization amounts to spontaneously reaching (for free) a critical angle and oscillating around this angle. Moreover, these dynamic sand piles are dissipative-morphogenetic processes, but they are not inherently dissipative: the sand pile stabilizes if the flux of energy/matter stops; particularly, this distinguishes them from the autopoietic and homeorhetic phenomena essential to life. Nor does there seem to be a global coherence structure or a global correlation length: the arrival of a new sand grain produces avalanches at all scales; a very interesting phenomenon, but one that is insufficient to develop the views on life we will focus on. As a matter of fact, the activities of an organism are surely not scale invariant: different levels of organization (cells, tissues, organs...) are understood by different mathematics, if any, as they yield different causal structures, strongly correlated though by integration and regulation.

In other more complex examples however, it is interesting to note that the formation rules can change over time and do so according to the characteristics of the system: the dynamic is "adaptive" [Solé, Goodwin, 2000].

In general, the physics of criticality enable to address many cases where interactions matter more than individuals. Now, there is no doubt that biology (and cognition) are above all an issue of interactions, of formations of organized (coherent) structures that are relatively stable. Moreover, critical thresholds spring up everywhere: from genetic mutations, which propagate only from a certain probabilistic critical threshold, to the action potentials of neurons. Likewise, one can analyze many cerebral activities in terms of attractors. Coherent structures then appear beyond the "edge of chaos", in specifically chaotic situations, where these attractors form and disappear dynamically, in an excitable environment. The slightest perturbation can entail the passage of a critical threshold, typically at the edge of chaos, which triggers a spate of different activities, and the theory of criticality can also provide us with information on this. Is it that the methods of physics suffice to make these aspects of living phenomena intelligible?

We believe they do, in part, because they provide a new and interesting venue for the analyses of the complexity of life and of its "physical singularity". However, the framework remains completely internal to physics and not a single one of the examples in the literature requires different tools than those used for inert structures, maybe with the exception of Kauffman's correlated landscapes, to which we will return. In fact, most of the sufficiently mathematized examples are of a physical nature or represent only the "physical friction" of living phenomena with its interior or with its environment: an essential component of its being in the world, albeit highly incomplete. Let's try to explain this by means of an 
example. The fractal structure of many organs (lungs, vascular systems...) is the result of a problem of optimality (maximize the exchange of energy through a surface, in a volume...) where the physical structure forms in presence of a critical threshold. The growth of living organisms is essential for this, but the interaction is physical: similar optimal structures can be reproduced with inert materials (beeswax, the fractality present in many physical structures or digital simulations...). These structures are "geodesics" in the appropriate phase space (parameters and observables), as is the case for any physical evolution; one of their main characteristics resides in the punctuality of the critical passages, as the critical values of the parameters are mathematical points. All phyllotaxis, with its very interesting and highly developed mathematics, represents this friction between the thrust of life and the physical environment. It always describes, and this is no coincidence, phenomena at the level of organs, which are the locus of the exchanges, of energy in particular, between the living organism and the physical environment (see [Bailly, Longo, 2003]). Once more, the use of the physics of criticality by Bak, Kauffman, etc, is of great interest. It gives a possible insight into the emergence of life from the inert [Kauffman, 1995]. It enriches the analysis of Darwinian evolution, rooted in selection, by a motor that it was lacking: the construction of order from disorder, on the basis of solely physical considerations; let's mention, for example, the networks (metabolic, informational...) which stabilize themselves beyond a certain threshold, or after the emergence of structurally stable correlations... In fact (and the demonstrations of this are now numerous), the randomness of mutations is insufficient for understanding phylogenesis: the spontaneous formation of order, "order for free", provides a fascinating (and relevant) alternative - an order which is then submitted to selection, of course.

These analyses, however, stop at the "threshold of physics": they bring us to the "edge of chaos", which remains a punctual transition, as everywhere in physics (a very specific value of the control parameter). This punctuality is quite necessary to the physico-mathematical methods widely used in this field: the renormalization group. One of the most telling examples in this regard, percolation [Solé, Goodwin, 2000], is interesting precisely and especially for the punctuality of the critical transition; but this punctuality is very general, see [Solé, Goodwin, 2000]. The modest number of parameters, sometimes a single one, according to which criticality is examined, is also an essential aspect (sometimes said to be positive) of its approaches and of its renormalization methods. The formation of life itself would then be a critical transition, with regards to one or two parameters; and life would situate itself, with its specific phenomena, within a zone that is close, but beyond the critical threshold.

Now, from our point of view, the physical singularity of the phenomena of life consists, among other things, in the robustness of criticality, which tolerates an extension of the critical passage. The punctual "edge of chaos" of physical analyses no longer appears to be sufficient: it must transform itself into an interval relatively to all relevant parameters (very numerous: time-space, temperature, pressure...). In a certain sense, the works mentioned attempt to make intelligible the formation of life, from the inert, as critical passage, but they do not examine as such the lasting phenomenon of life, its "extended criticality". Let's also mention that the conceptual singularity of life also resides in the dynamic of the phase space. We will return to this.

To summarize, the mathematical challenge, with regard to current physico-mathematical theories, consists in the non-punctuality of the structural stability of life (extended criticality relative to numerous control parameters) as well as in the difficulty of establishing a fixed landscape (phase space), within which any process would unfold, following geodesics 
punctuated by critical transitions. In this regard, it is the phase space itself which changes dynamically (a new organ, a species - unexpected observables and parameters - grow during the course of ontogenesis, of phylogenesis): the dynamic can also be found in the very observables and parameters of the ecosystem, a coevolutive framework where the emergence of novelty changes the basic situation. Kauffman's notion of "correlated landscapes", with their dynamics, and "adaptive dynamics" [Solé, Goodwin, 2000], are close to the idea we mention in this text; they are quite rich, particularly from the mathematical viewpoint, because the first, for example, specifies a very interesting notion of "correlation" within a phase space normally considered as governed by randomness. They appear however to force a conceptual and mathematical stability, specific to physical theories, since the space of parameters and of observables is given a priori. This is insufficient in our opinion to grasp the evolutive dynamics of living phenomena (see below).

On the other hand, within these changing evolutive spaces, what is relatively stable and robust is the lasting or extended criticality of the living object, in contrast to the singularity of criticality in physics: as long as it remains within a range of possibilities (by adapting) the living individual survives along a generic path (a possible, but not necessarily optimal one) within spaces (ecosystems) that are intrinsically changing. Our conceptual outline therefore locates itself at the limit of the physical theories of criticality and attempts to grasp certain aspects of the phenomenality specific to life, in the hope that a unity (or... a critical transition) may then establish itself with the concepts and, above of all, the mathematics of emergence and of physical self-organization. We finally observe that the notion of extended criticality, as developed here, was first hinted in [Bailly, 1991], whose scientific reference goes back to the early work on self-organized non-equilibrium systems, summarized in [Nicolis, Prigogine, 1977], more than to the approach by the authors above (see also the end of the next section, where we further stress the difference).

\section{Life as "extended critical situation".}

We have seen that a critical state, in physics, is a singularity within a process: that is, that all along the process, a position, a configuration, a "state" which may be assumed briefly before the global state of that which we are observing changes (radically). A critical state can also be seen as a bifurcation or, in certain cases, as a catastrophe, in the sense of Thom, particularly if this change becomes irreversible. In a sense, it is the opposite of a situation of equilibrium (opposite sign in the mathematical description); and it differs also from the notion of "being far from equilibrium", since this situation, generally, does not imply "possible different evolutions" of the system (bifurcations).

We consider life phenomena as being far from equilibrium and continually submitted to perturbations. Thus, by definition, in current physical theories, these systems cannot remain "for long" in a critical state: the development over the course of time, or the intended parameter, forces it beyond that state; this is the pointwise nature of criticality. Also, in Physics, criticality is a typical aspect of transition, where minor fluctuations, possibly below the level of observability, can lead to radically different evolutions. The instantaneous nature of criticality has been very well expressed in mathematics by the divergence (towards the infinite, see previous) of functional descriptions, according to specific parameters. Or also, as we have previously discussed, by the maximum of complexity, which also creates instability. 
In contrast to physical situations, it very well seems that life, which we may represent, at least for a certain duration, as a stationary state far from equilibrium, evolves within an "extended critical zone", which lasts over time and of which the criticality could be represented as a dense, even continuous, set of critical points in the phase space. This trait would be made possible by the spatial organizational enclosure of an organism, by its finitude in time and by its homeostatic autonomy (integration, regulation). Also, as we have highlighted elsewhere in ore details, [Bailly, Longo, 2008], living matter is characterized by the coexistence and the articulation of several types of temporality (cycles for internal clocks, relaxations for the phenomena of stimulus/response, internal or external). Corresponding to it is also a hierarchy entangled with levels of organization of differing natures (biolons and orgons ${ }^{4}$ ) and the passing from one level to another is comparable only at a conceptual level (though a useful comparison, we believe) with that which operates in physics, by means of renormalization for instance, between the local and the global in a given critical point: there exists characteristics intrinsically different amongst a same system depending on levels and scales. This requires the introduction of concepts beyond those of physics, such as, we have seen, those of integration and regulation, coupled to that of biological function on the one hand, of contingent finality or of anticipation on the other (which at the same time modifies the causality regime of living organisms with regard to that of physics) ${ }^{5}$.

Our thesis, therefore, is that the notion of physical criticality could help us to understand a biological situation as a physical singularity "of long duration", "an extended critical situation", in which, particularly, homeorhetic processes maintain a permanent tension between the local and the global. A physical situation which, mathematically, is understood as the locus of a divergence, of a passing from the finite to the infinite, and as the singular point where the relevant object changes (renormalization, see note): the local is integrated into a new object, the global object (the living unit or biolon). Thus, in physical terms, the measurement of the objective complexity of the slightest of living units, a cell, a global structure and its components, has an infinite value, in a specific mathematical sense. Likewise, if we consider physical parameters as for measurements (length of correlation and its relative effects), the living element is in a progressive critical situation, a permanent passing between the local and global. It is thus that a singular dynamic unit, that of living matter, from the cell, is infinitely more complex than any physical process, which may

\footnotetext{
${ }^{4}$ Synthetically, we call a living entity biolon (a cell, an individual, animal or vegetal, an entire species). Biolons are composed of orgons (the organelles of a cell, the organs of an individual, the organized populations of a species). This terminological unification is justified by the uniformity of concepts, of mathematical tools, with which one can address the three levels brought together under the same name (see [Bailly and al., 1993] and [Bailly, Longo, 2003], where the notion of extended criticality is just hinted).

${ }^{5}$ An image probably enables to better illustrate what we mean by that : if we represent the efficient causality of physics as an arrow extending from an initial point to an final point, on a line, the line would be infinite and would thus form a non-compact support; biological causality, which would add itself to physical causality, could then be represented by a same arrow but on a closed line, this time, forming a compact support, representing the effects of retroaction and of finalization (possibly also related to the effects of enclosure - and of autonomy - which have been evoked). Passing to another level of analysis, we could consider that this type of causal manifestation takes place on the internal fibers constituting additional compactified dimensions. The two other causal structures are of course compatible and "simultaneous", because living phenomena is immerged within physical fields.
} 
behave in a critical fashion only in exceptional cases, of short duration, as mathematical singularities.

To summarize, a unit of living matter, a biolon according to our terminology, critically instable, is preserved in its extended situation, far from equilibrium, by homeostasis, or better, by homeorhesis. Or else, the dynamic integration and the regulation of its components (orgons, with their components, biolons, with their orgons...), their "agoantagonistic" relationships [Bernard-Weil, 2002] within themselves and their environment, sustain them within an improbable physical state. Autopoiesis, [Varela, 1989 ; Bourgine, Stewart, 2004], constitutes another way of expressing this auto-constitutive dynamic (see sect. 2.3 below). A mathematical organization which may be related to autopoiesis could refer to several coupled endomorphisms, in mathematical terms; their "organizational enclosure" may then correspond to the limits of structurally stable attractors; the insides and the membranes may be understood respectively as the basins and the edges of attractors (or, to be more precise, as the physical expression of the proper attractors, which are given in the phase space).

From the moment that integration or regulation no longer works or exceeds the limits of the pathologically tolerable situation (limit of functional plasticity), everything collapses: entropy suddenly grows, disorder represents death. Running upon a tight rope is a good representation of the progressive situation of a biolon: when control, as regulation and integration, decreases up to a certain level (the critical boundaries of the extended critical situation, as acceptable limits of pathology) death terminates this contingent life, by a final and irreversible transition state. Of course, at the boundaries of the extended critical situation, phase transitions, changes in correlation length, passage through singularities... continually occur, but, within the considered limits, they are confronted to a regulating activity. In fact, they form an essential part of it: all biochemical thresholds which contribute to a biolon's internal exchanges may be perceived as elementary components of global homeorhesis. More globally, life itself can be seen as an "extended physical singularity".

Physical paradigms have helped us to formulate this notion, which is not of a physical nature. Monism, intrinsic to modern science, refers to matter, and not to methodology; in fact, we face different phenomenalities and attempt to make them intelligible by organizing them by means of various notions and conceptual structures (and, if possible, mathematical ones). A synthesis is far from being obvious; it must be constructed by means of a new (and mathematical) conceptual unity, as long term objective.

As we have already discussed, the formation of such an extended critical zone, associated to the conditions of possibility for life, could proceed from the convergence of two processes whose origins are opposed: on one side (first boundary of the zone), following [Nicolis, Prigogine, 1992]), starting from equilibrium, a series of critical bifurcations which could be generated by fluctuations while moving away from equilibrium and, on the other side (second boundary of the zone), the self-organized criticality stemming from the stabilization of originally chaotic states (see [Kauffman, 1993; 1995], [Goodwin et al., 1992] ${ }^{6}$ ). In short,

\footnotetext{
${ }^{6}$ As for the approach in [Bak et al., 1988], observe that extended critical situation can only exist and maintain itself far from thermodynamic equilibrium and in the active presence of exchanges of matter, energy and information with the environment. In this, it comes close to the conditions within which dissipative structures constitute themselves while at the same time distinguishing itself from self-organized criticality (examplified, namely, by the behavior of sand piles) such as has been specifically studied in [Bak et al., 1988]. As we mentioned above, the latter are a dissipative-morphogenetic processes, but they are not inherently dissipative (the sand pile stabilizes if the flux of energy/matter stops) nor yield coherence structures.
} 
we consider that both approaches, ranging say from Prigogine to Kauffman, are extremely interesting, yet, we believe that each of them describes only "one side" (one "conceptual boundary") of the phenomenal situation of life: in our approach, they coexist and bound the extended criticality of living systems, in the space of parameters. Moreover, the possibility (and constraint) of the coexistence of these two different boundaries would be precisely associated to:

- the formation of levels of organization interacting between themselves,

- the organizational enclosure of autopoiesis,

- the constitution of a homeostatic autonomy capable to maintain itself ${ }^{7}$.

\subsection{Extended critical situations: general approaches.}

What can it mean, for a system, to have to evolve within an "extended critical zone"?

First, it must be to find itself at all times far from equilibrium because the maintaining of its organization requires intensive exchanges of energy allowing to maintain an "abnormally" low entropy with regard to the situation of equilibrium.

Next, and correlatively, to present itself with an internal organization, that can extend to the constitution of levels of organization, corresponding to the constraints of dissipation (dissipative structures) within the context of these exchanges.

Third, to form a "whole" within time and space (at least locally and momentarily), inasmuch as its internal correlation lengths are of the size of the system itself (critical situation) and its characteristic times, of more or less exhaustive running through the attractor corresponding to its situation, are bounded, thus defining the scale of its existence (namely by the maintaining of its internal organization). In this way, therefore, is manifested a first from of spatio-temporal autonomy, coupled to this fundamental heteronomy which constitutes the previously highlighted necessity of exchanges with what lies beyond. In this, also, the system presents itself as an "extended" singularity in the usual physicochemical landscape (possibly calling for a theory of generalized catastrophes, an extension of the theory of elementary catastrophes).

Does evolving in such a zone impose the existence of an organizational enclosure and the establishment of a boundary? An argument in this direction would be related to the necessity of limiting leakages and of regulating the exchanges of energy; another, within the framework of an autopoiesis, would be related to the necessity of implementing boundary conditions enabling the very existence of such a system; a third could be found in the fact that the existence of an extended critical zone would only be possible upon a topology enabling to distinguish an inside from an outside (compacity argument?). These arguments are somewhat circular inasmuch as one supposes the existence of the system in order to define the conditions for its existence. This must signify that the hypothesis which consists in associating living phenomena to a zone of extended criticality, if it enables to interpret the

\footnotetext{
${ }^{7}$ We know that in physics it is the boundary conditions which generate (for vibrating strings, for instance) the discrete stationary states that are solutions to propagation equations. Maybe can we find a metaphor for the constitution of these levels of biological organization related to both the constraints of organizational enclosure as well as to those of having to evolve within an extended critical situation bounded by the characteristics which we have just evoked (sequences of bifurcations, on one side, chaos, on the other).
} 
permanence and the functioning of organisms, still remains insufficient to describe or to explain the origin. What appears, at this stage, namely by the fact that this hypothesis says nothing about learning capacities - even if in certain regards the quest for structural stability can somewhat resemble them -, and especially nothing concerning anticipatory capacities (or contingent finality) - even if the spontaneous implementation of a timescale characterizing its own limits of existence may be considered as a prefiguration of protension (a time scale may correspond to an "extended present", thus to the time of "protension", which assumes learning, see [Varela, 1999]). That is, life cycles and rhythms may relate to Varela's Husserlian "extended or specious present" or to Rosen's anticipatory capacities, an issue to be further investigated.

In what concerns the objectivity of these constructions, it is not only an issue of distinguishing within living phenomena itself aspects of structure (anatomy, for example) and dynamic aspects (physiological functions, for instance), but also of having particular ways of viewing living phenomena in general, either structurally (a-temporally), or dynamically, associated to genesis and duration (see, for example, the theory of viability [Aubin, 1991]).

Different approaches, no longer solely conceptual but also more or less mathematized have for a while now been distributed according to this partitioning, without their articulation having been solidly ensured, to our knowledge. Among the most recent, let's mention on the one hand the applications of the theory of singularities (elementary catastrophes) by René Thom and on the other hand the application of the thermodynamic theory of bifurcations by Gregoire Nicolis and Ilya Prigogine. One of the possible contributions should consist, for the structural aspect, in the recourse to a mathematization within the framework of Category Theory (also a-temporal) and, for the dynamic aspect, to develop a theorization of the extended criticality that we have just evoked by introducing the couplings and scales of relevant temporalities. The articulation of the two approaches could introduce the relevance of temporalities in the "categories" aspect and a stable genericity (see below) in the "extended criticality" approach.

But let's nevertheless try right now to be more specific and more precise in our attempts to characterize that which we mean by "extended critical situation".

\subsection{The extended critical situation: a few precisions and complements.}

\section{A. Spatial aspect: concerning correlation lengths.}

The "correlation lengths" for living matter seem to be closely related to the transportation properties of the various molecules and substances which actually enable "to pass information" from one locus to another, or to modify the biological behavior in one location from a stimulus originating from another one. Now, depending on the size of the biolon, there seems to be two types of transportation processes likely to occur. For larger organisms, that would be of the "propagative" type (velocity $v_{p}$ ) and the typical correlation length will be $\mathrm{L}_{\mathrm{p}}=\mathrm{v}_{\mathrm{p}} \tau$, where $\tau$ represents the characteristic amount of time. For small organisms (cells, for instance) we would have a "diffusive" type (diffusion coefficient D) and the typical correlation length would be $\mathrm{L}_{\mathrm{d}}=(\mathrm{D} \tau)^{1 / 2}$. (Neurons, of course, present the notable exception of "diffusing" also action potentials.) 
We can notice the difference in dependency according to time: linear in one case and in a $1 / 2$ exponent in the other.

Two complementary remarks:

- The size of the organism is also at play in the nature and the implementation of the selected structures to ensure the mode of transportation; for example, in the case of the respiratory function (transportation of oxygen) in small organisms (insects, for instance) the transportation takes place through tracheas (or pores), a multitude of little cylinders where the air is diffused in order to supply the cells with oxygen; in larger organisms (fish, mammals) the transportation and exchanges take place by means of gills or lungs, "centralized" anatomical structures presenting fractal geometries enabling to reconcile the hardly compatible constraints we have already mentioned (efficiency, stericity, homogeneity); the transportation, in this latter case is also much more of a type which we can qualify as "propagative" (even if diffusion does play a part for bronchioles, namely).

- These considerations are mainly valid for different structural aspects relating to identical functions. The functional aspect, on the other hand, is very generally governed by common scaling laws which we have already discussed (the metabolism which

corresponds to oxygen consumption, the variegated rhythmicities, the periods of relaxation,...). It thus appears that the modes of transportation associated to identical functions can differ and can correspond to different anatomical structures.

- Finally, account taken of these remarks, if the characteristic times $\tau$ generally scale as $\mathrm{W}_{\mathrm{f}}^{1 / 4}$, where $\mathrm{W}_{\mathrm{f}}$ corresponds to the average size (or mass) of the adult organism, one must expect the correlation lengths to scale differently according to the modes of transportation: respectively $\mathrm{L}_{\mathrm{p}}$ in $\mathrm{W}_{\mathrm{f}}^{1 / 4}$ and $\mathrm{L}_{\mathrm{d}}$ in $\mathrm{W}_{\mathrm{f}}^{1 / 8}$.

\section{B. Temporal aspect: return to the specificity of the temporalities of living matter.}

We outline here an idea developed elsewhere concerning biological temporalities ([Bailly, Longo, 2008]). The causal intrications of the various levels of organization of living matter have led us to think that the extended critical situation - corresponding to the self-referring and individuated character of the organism - presents a topological temporality of the $\mathbf{R x} \mathbf{S}_{\mathbf{1}}$ type, where $\mathbf{S}_{\mathbf{1}}$ is circle. Thus, $\mathbf{R x} \mathbf{S}_{\mathbf{1}}$ is the continuous time of the real straight line times a compactified time-line, a circle, $\mathbf{S}_{\mathbf{1}}$ - to be naively understood as measured by an angle, as on a clock. This is the internal time, where one may represent the internal clocks, while the organism's externality (and the way in which this externality reacts with the organism) preserves its usual temporal topology R. The idea is to provide a "reference system" (the "axes for representation, in the Cartesian sense) accomodating physcial time and bilogical rhythms (see [Rossi et al., 1992], say, or [Bailly, Longo, 2008], for more references). Thus, from the standpoint of time now, the extended critical situation would be characterized by this bi-dimensionality, being coupled, from the spatial standpoint, with quasi-infinite correlation lengths (at the scale of the whole organism) and the organizational enclosure would conjugate these specific spatiotemporal aspects which would manifest as autopoiesis. The extended critical situation would therefore delimitate, from the standpoint of spacetime (to which we will add all other relevant parameters such as temperature, pressure, the quantity of nutrients or of oxygen,... see next), a sphere of a spatial radius $L$ for instance, where the correlation lengths of the interactions would be of the order of $\mathrm{L}$, and the "temporal radius" $\tau_{\mathrm{v}}$ (lifespan within $\mathrm{t}$ ) $\mathrm{x} \theta$ (varied cycles). 
Without changing the essence of the question, we can nevertheless present a slightly different way of seeing it: for a living organism, the extended critical situation would occupy a volume within an n-dimensioned "space". Among these $\mathrm{n}$ dimensions, we could distinguish three spatial dimensions as such ( $\mathbf{R}^{\mathbf{3}}$ topology) and two temporal dimensions ( $\mathbf{R x} \mathbf{S}_{\mathbf{1}}$ topology) of which the compactified dimension takes a non-null radius outside of this volume, the remaining $\mathrm{n}-5$ volumes corresponding to the compatible values of the vital parameters (temperatures between $T_{1}$ and $T_{2}$, metabolisms between $R_{1}$ and $R_{2}$, etc.). The metrics of the volume space maximally correspond to the life-spans (for $\mathbf{R}$ ) and to the pure maximal numbers (maximal endogenous frequencies) for $\mathbf{S}_{\mathbf{1}}$ (for more on this, we refer the reader to [Bailly, Longo, 2008]).

One will notice that the endogenous rythmicities and cyclicities are not rhythms and cycles (whose dimension would be an inverse of time), but iterations of which the total number is fixed regardless of the empirical life-span, at least in metazoan.

\subsection{More on the relations to Autopoiesis.}

Our conceptual frame may also be understood as a way to further enrich and specify the notion of Autopoiesis ([Varela et al., 1974]). Autopoiesis doesn't imply extended criticality, nor the other way round, but the two notions together may join in an effort to focus on key phenomenal properties of living systems. Recall that an autopoietic structure is

" a network of processes of production (transformation and destruction) of components which: (i) through their interactions and transformations continuously regenerate and realize the network of processes (relations) that produced them; and (ii) constitute it (the structure), as a concrete unity in space in which they (the components) exist by specifying the topological domain of its realization as such a network. "

Thus, autopoiesis may be seen as a way by which the living generates and preserves extended criticality, far from equilibrium, yet in a functionally coherent structure. The qualitatively constant internal environment (in relation to the external environment ) is part of structural stability or stability of the qualitative organization as captured by autopoiesis and departs from cybernetic feedbacks or alike. Autopoiesis contrasts the increase of entropy due to the metabolic/thermodynamic irreversible processes, by improving/maintaining the organization (a growth/preservation of organization decreases the corresponding entropy). And this is a crucial "physical singularity" of living systems: the capability to lower entropy, along an irreversible process, by (re-) constructing organization.

As already observed, both autopoiesis and extended criticality require an "operational closure", that is a delimited space-time for the evolution of the process. Extended criticality proposes it by the (limits of the) coherence structure proper to critical states (due to correlation lengths). The peculiar dynamics of autopoiesis may be understood in the terms of the local unstability of criticality, which we also described as a dense set of critical points in the phase space associated with a global structural stability, even limited in space and time: an autopoietic system continually (and locally) moves from an unstable state to another, while preserving the global coherence (or structural stability). This feature may help to understand of its ability to explore various local surroundings (ecosystems) and to adapt to new (internal or external) constraints which appears as plasticity properties. We will go back to autopoiesis in the Intermezzo below.

\subsection{Summary of the characteristics of the extended critical situation.}


To summarize certain aspects encountered and discussed here, we could characterize the extended critical situation namely by means of the following (non-exhaustive) traits:

- a spatial volume enclosed within a semi-permeable membrane

- correlation lengths of the order of magnitude of the greatest length of this volume

- a temporal bi-dimensionality, one classical, bounded between conception and death, associated to the bio-physicochemical evolution of the organism in relation to an environment (behavior which can be of the relaxation or of the cyclical types), and the other compactified, associated to the endogenous physiological rhythms of the organisms, manifested by dimensionless numerical quantities

- a metabolic activity far from equilibrium and irreversible, involving exchanges of energy, of matter, of entropy with the outside as well as the production of entropy

- a confinement within a non-null volume of a space of parameters (temperature, etc.) of $\mathrm{n}$ dimensions

- an anatomico-functional structuration into levels of organizations which are autonomous, but coupled amongst themselves, likely to be distinguished by the existence of fractal geometries (membranal or arborescent); the fractal geometries can be considered as the trace (or model) of effective passage to the infinite limit of an intensive magnitude of the system. These levels of organization alternate biolons and orgons (the latter being essentially the locus of manifestation for fractal geometries). The lengths of correlation manifest both within and between these levels.

\section{Integration, regulation, and causal regimes.}

A few additional facts may also relate physical and biological phenomena, while also highlighting their differences. Causal relationships are local in physics; they may be global only in the sense of a field connecting physical entities by the propagation of local interactions. Thus, in this approach, the global structure, with its correlation length, is only obtained by means of the transitivity of local interactions. This way, in each of the physical cases (local/global), mathematics, by fixing a phase space, isolates a unique level of causality. In biology, conversely, the local causality may radically differ from global correlations, and yet cannot be isolated from the latter: integration and regulation, typically, causally affect local interactions (local biochemical exchanges can be regulated by hormonal cascades or by neural signals of a completely different nature). In other words, the global causal regime may differ from the local regimes, even if they permanently interfere with one another: thus, the unity of a biolon is given by a regulatory-integrative activity, of a different physical/biochemical nature with regard to the exchanges between and within orgons, although that these exchanges are regulated (and affected) by the global. Notice that it is the global regime that essentially contributes to homeorhesis (autopoiesis, ago-antagonistic couplings) as the maintaining of an extended critical situation; its fluctuations, within the boundaries of criticality, correspond to the pathologies that a biolon may live with, as well as to some forms of local death (cellular apoptosis within an orgon). Naturally, fluctuations exist in physics (and can be "tolerated"), but the underlying physical entities do not change over the course of the fluctuations. In this sense, in physical theories, there is nothing that resembles phenomena such as pathologies or local apoptosis; in that, the latter's mathematics still remain to be invented, as much as their pre-formal conceptualization. 
To summarize, integration is the presence (upwards-causally) of the local within the global structure, whereas regulation is the global structure causally affecting (downwards) local structures. It is this local/global interaction which organizes the "internal conduct" and which maintains the extended critical situation at least at the phenomenal level (see also [Rosen, 1991; Stewart, 2002]). But besides having this role of stabilization, these interactions also participate in what we will later define as a (possible) theoretical indetermination of the evolution of living matter (both along phylogenesis and ontogenesis), because they interconnect different levels of organization, each governed by its own regime of physical causality: we will then also attribute indetermination to the "resonances" between these various causal structures.

Despite the physical singularity of living phenomena, let's attempt once more to define our understanding in familiar physical terms. Regulation may play the role of initial boundary conditions for the global behavior of the solutions of systems of equations (differential or of finite differences), that is, dynamic systems described by these equations. Integration may also be understood, by rough analogy, as the correlations of variables conferring unity to a given system of equations; or, also, in its role of organizing singularities in their solutions. It may also be understood, likewise, as the analytical extension of a locally defined solution. Once more, we are only attempting to approach, by means of physico-mathematical concepts a phenomenology which, till now, extends far beyond these descriptions, in the mathematical sense of an infinite complexity, defined in $\S .1$ and 2 (the complexity of a singularity).

\section{Intermezzo: on contingent finality and entropy.}

Inter1 : Finalism.

In our view, biological causality needs to integrate some notion of finalism, in a theoretically autonomous approach to living systems. When and if reduction to physical theories will be fully accomplished, then (a suitable) physical causality, possibly timeoriented, will surely "explain" in more appropriate terms, the various circularities of life, including the causal ones. But this may require a change or an enrichment of current physical theories as much as quantum mechanics and relativity are ongoing deep revisions towards unification (of space and time - non-commutative geometry - or of the very objects - string theories). In the meanwhile, for our theoretical purposes, we called "contingent" the conceptually needed finalism of life as :

- it doesn't need to be there (it is not necessary : life or a specific living being may not exist nor last) ;

- it is local not global, in a sense we will try to explain next.

The natural way to see finalism in life is given by anticipation or protension, in phenomenological terms : an anticipated event causes or influences an ongoing process. More deeply or at the simplest level, a metabolic cycle tends towards its own preservation, when it takes place in a living organism - a basic form of protension. Again, this may be seen as part of the definition of life : life exists exactly because it tends towards preserving itself - otherwise it would simply not be there - this is its contingent finality.

In this sense, autopoiesis may be seen as a contingently finalistic process : the interacting network of processes tends towards the productions of the components that produce the processes. Otherwise, since it is far from equilibrium, as soon as the "effort" of maintaining itself in a permanent flow of energy stops, it would collapse in that state of 
equilibrium which is called death. Autopoiesis is a way of describing the maintaining and/or improvement of metabolism, which is the (minimal and) contingent finality of life.

In contrast to old fashioned leibnizian finalism, we are aware that causality in modern physics is largely analyzed in terms of "symmetries" and symmetry breaking (see [Bailly, Longo, 2006; chap. 4] for a recent account). In particular, symmetries correspond to conservation properties, thus they realize the geodesic principle in various contexts. In the theoretical analysis of life, it seems that the addition of a further conservation principle is required (or useful): "preserving autopoiesis" (that is life itself). By its nature, this may seem finalistic and may break physical symmetries. In particular, the geodesic principle doesn't seem to govern the directions of evolutions in so far these are only possible (generic) paths: the phylogenetic (and ontogenetic) drift, [Maturana, Mpdozis, 2000], goes along all possible (compatible) directions, it does not follow a (unique) geodesic. One more reason to consider insufficient a causal analysis based only on symmetries and geodesics: the finalistic preservation of autopoiesis (and thus metabolism) "forces" species and living objects to go along all possible evolutive paths.

Inter2 : Entropy.

Let's now further argue on the local nature of this new conservation principle that life implements, in reference to entropy. In our view, the relevant property of autopoiesis is that not only it produces networks that produce components that produce those networks, but also that it contrasts the inevitable growth of entropy related to the intended and irreversible flow of matter and energy. As a matter of fact, there are two kinds of entropies that participate to the process. On one side, the thermodynamic production of entropy related to irreversible energy consumption, an entropy which, by this, continually grows [Nicolis, Prigogine, 1992]. On the other, autopoiesis decreases entropy by increasing or maintaining organisation. Organization, thus negative entropy, increases at least during embryogenesis; later, it is maintained, though ageing may slow the (re-)organization process. Death supervenes when the (re-)organization process cannot oppose anymore or not sufficiently, by decreasing entropy, the increase of the other kinds of entropy.

In short, on one side, entropy grows as a consequence of irreversible physical processes. On the other, entropy decreases by a permanent (re-)organization of the living material (formation of tissues and organs first, their maintain and permanent renewal later). Now, this contrasting productions of entropic processes manifests itself locally : at each instant autopoiesis produces enough organization as to prevail, in the game of entropies. More or enough organization is produced as to continue the game, unless pathologies modify the dynamics or lead to death. However, this very game is an irreversible process, it thus produces its own entropy. And death is the inevitable end of it. Yet, and this really matters to us now, there is no need to propose a global finalism to understand autopoiesis: as we said, at each instant autopoiesis goes along the contrasting line of entropies, and a local analysis of its evolution makes it understandable.

This understanding is not so different from the turn that departed physics from finalism, at the beginning of the XIXth century. Till then, light was seen to go along the shortest path, since it were following the best way to go towards an objective. Similarly, inertial movement was often described in finalistic terms: the choice of a geodesic or of the optimal trajectory in order to... go somewhere. The situation radically changed by an understanding of geodesics as (mathematical) integral (a sum) of local properties. To put it briefly, a straight line is defined locally by points where the tangent is constant. Similarly, conservation of energy or of other quantities is a local, point by point, property (galilean 
inertia is a local): the global path is obtained as an integral (a sum) of the local values. Thus, finalism in physics was put aside as inertia, typically, or "going straigth" or along a geodesic is the result of a previous or present history of the phase space and nothing else.

We are not (yet ?) able to perform this in the analysis of life and we need to integrate in our theoretical proposals the contingent (local) propension to contrast the growth of thermodynamical entropy (due to the irreversible flow of energy) by the decreasing entropy of a permanent (re-)organisation of living structures. Yet, we see this as a local form of finalism, which realizes itself instant by instant and does not need to assume any global, preestablished finality ${ }^{8}$.

\section{Phase spaces and their trajectories.}

Biological situations would nevertheless present an additional trait, rather essential in our view: as we have already emphasized, this spatio-temporal extension not only concerns the behavior of "trajectories" within a given phase space, but also reveals itself through a modification, through living phenomena, from this phase space (ecosystem, in a very broad sense) itself. These modifications would concern the interaction with the environment and would be induced by mutations, the apparition or disappearance of certain organs, even of populations, etc. The situation would be rather comparable to the existence (metaphorically speaking) of a sort of set of phase spaces (the one corresponding to the union of all possibilities for a given organism), characterized only by a few great structural invariants (we are thinking here, namely, of the absolute numbers which transversally characterize many expressions of the living phenomena, such as metabolic rhythms, for instance). These great spaces of invariants would be themselves partitioned into phase (sub-)spaces (those corresponding to the effective coming into being of these possibilities), the trajectories generally taking place within these sub-spaces along strange attractors. Yet, they may be likely, under certain perturbations (genetic mutations, brutal change of physicochemical or biological environment), to pass from one sub-space to another, thanks to the properties of these attractors (see §.5.1).

However, this set of phase spaces (or of evolutions) would be far from being predetermined. More specifically, it is at this level, firstly, that one should locate a sort of biological indetermination: that is, at the level of the passage from one phase space to another (ecosystem), at a given moment, to that of the following moment; and this passage

\footnotetext{
8 A paradigmatic case of global finalism is the theory of programming in genetics (see [Longo, Tendero, 2006]): if the genome were a program, in the sense of Computer Science, each cell of a metazoan would contain a well written code for all its phenotypic aims in life. Programming is the most finalistic activity that man ever invented: a program is written for a possibly remote aim. In contrast to this view, we understand genes as traces of a phylogenetic history, which intervene in the autopoietic process by statistical, stereospecific interactions with macro-molecules. By their rigid structure they contribute to set the limits of extended criticality. In particular, they induce the primary structure of proteins as well as, indirectly and by epigenetic phenomena, the proteins' shape - secondary and tertiary structure. In short, genes force the approximate iteration of an history; thus, they contribute to the species' structural stability, in conjunction to the relative co-constituted stability of the ecosystem, by "forming" the molecular material of autopoiesis. Yet, by the genetic drift and degenerescence, in the sense of Edelman (see [Bailly, Longo, 2006]), they also contribute to variability.
} 
would "contain" or would express the biological trajectory (phylogenetic, ontogenetic). To explain this, let's briefly return to the analogies/differences with physics.

In classical physics, a phase space is given within which form the specific trajectories, actually the geodesics of the relevant objects (see $\S .5$, further down); in some cases (nonlinear, typically) the formal global determination of these evolutions (the existence of equations determining the dynamics) does not necessarily imply the predictability of the trajectories (but the system remains deterministic). On the other hand, in quantum physics, once the phase space is given, (the quantum states within a Hilbert space), the dynamics does not go along proper space-time trajectories, in the classical sense, and it is an intrinsic indetermination which governs the theoretical intelligibility of the phenomena; particularly, the causal relationships are replaced, within the given phase space, by correlations of probability. This conceptual and mathematical framework, with its indetermination and its probabilistic analyses, is at the centre of quantum mechanic's theoretical originality (and of its conceptual difficulty).

We believe instead that, in biology, the theoretical and conceptual difficulty resides principally in the impossibility to give, upwards (or a priori), a formal global determination and a phase space (or a space of evolutions) able to enframe the phenomena for a sufficient amount of time; that is, to describe a global mathematical determination (a set of equations, typically) within a phase space that is a priori and given once and for all, or at least for very long. In fact, if there exists a relative structural stability, it concerns the objects and some of their components, more than their framework of living, including their ecosystems or spaces of relevant observables and variables.

To put it in other words, in physics, the mathematical notion of "State-Determined Dynamical System" (typically expressed as a set of differential equations) does sterling service. Its operational effectiveness stems in large part from the fact that the dynamical laws are fixed, i.e. they are "laws of nature" or "conceptual construction principles" that are given a priori, within the theoretical proposal, and it would be meta-physical to ask "why" they have this or that particular form. In addition, for a given system, the list of variables and the boundary conditions are also givens (at worst, in Quantum Physics, in presence of the "creation" of a new particle, one gives a Focks space in order to accomodate it, a priori, instead of an Hilbert space). The great difficulty is that, in biology, variables, observables, boundary conditions and even the dynamical laws themselves are actually constituted by the operation of the living organism itself. And this difficulty is multiplied yet again when we wish to take properly into account the interactions between an organism and its environment: because what the environment is also constituted by the organism, typicaly by its "ecological niche". Thus, there is through and through a co-constitution of the very frame of intelligibility. In conclusion, the "bifurcations" take place as much at the level of the selection between possible determinations, as within possible and different determinations.

Let's return to this delicate point, as it happens, in terms of trajectories. In mathematics, the critical points on a curve (maxima and minima, for example), may be said to be specific in that they are in most cases much less numerous (a denumerable discrete) than the continuous to which all the other points belong, which can then be said to be generic.

In physics, in this regard, within the given phase space, the set of "conceivable" trajectories is generic, while the effective trajectory, defined by the geodesic principle, is specific (critical, stable, meaning minimal for the Lagrangian action, or in the particular case of optics, minimal for the optical path). In other words, effective physical phenomenality is specific and is enframed within a relevant phase space (a great part of the physicist's job is 
actually to characterize this space). It is doubtlessly this which confers to physical theory a great mathematical force as well as a possibility, by means of abstraction, to characterize the physical objects using very general properties, despite the singularity of each specific experience of which the conditions are not always exactly reproducible: the trajectory of any object will be specific and its analysis is related to the phase space (abstract and general).

In contrast, it appears that for biology, the cells of an organism, the organisms of a species, the species of an environment (that is, the biolons), are concerned by "generic trajectories": all hose which remain compatible with their perduration, even their transformations (mutations). It would be the falling back upon the specificity of this generic which would cause it to lose its biological character each time a physical reduction is attempted.

From then on, it is possible to understand the extended critical situation as the expression of this genericity in contrast with the localized critical transition of physics (within the space of parameters and within time, due to their incessant fluctuations). To put it in other words, there would be a sort of duality between physics and biology: specificity of the trajectories and "locality" of the criticality for physics, genericity of trajectories and extension of criticality for biology. At the same time that would enable to "naturally" relate the biological to the considerable variability of which it is the locus given that this generic compatibility would allow for the existence of a great number of possible "trajectories". Invariants should no longer then be defined within a given phase space but over the set of phase spaces that are compatible with this genericity, each specificity being able to modify the local structure of this set without however disappearing. This is maybe what would explain the great invariants (approximated) which we have mentioned, almost always concerning considerable sets of organisms (mammals, oxygen metabolisms, the animal kingdom itself, etc.), because they would constitute some of the rare invariants with regard to this global plasticity. These invariants endure, within the variability of living phenomena, and contribute to its stabilization; moreover, this biological variability differs from physical variability, because the latter does not normally contribute to stability (at least in non-linear cases) and finds itself within a framework of determination, while the former may go beyond it.

To conclude, we believe that, for biology, it is the collection itself of relevant objects and of parameters (phase space) which will follow the current situation, which is indeterminate and that this indetermination should be intrinsic to the theory, as the co-constitution of living individual/species (biolon) with and within an ecosystem is a major theoretical challenge in biology. Within this changing frame, the trajectories themselves (notion that is preserved although it becomes problematic also in quantum physics), differ from those of classical physics, because they are not specific (geodesics within given spaces), but generic (possibilities within changing spaces). In a sense, there would therefore be two levels of inter-correlated indetermination: that of the passing into the new ecosystem and that of the "choice" of trajectory for each biolon, among all compatible trajectories. Over the course of phylogenesis, typically, the formation of a new species which participates to the ecosystem, even to the emergence of a new organ, modifies the evolutionary space, even the space of ontogenesis, and modifies the set of possible trajectories. Once again, the genericity of the trajectory ought not to be analyzed only within a same phase space, but also in terms of passage to a new possible space. 
For this reason, in our opinion, the analysis of the dynamics of living phenomena can not be reduced to the terms of classical deterministic unpredictability (with its geodesics, of which the choice is more or less sensitive to border conditions), but to those of an intrinsic indetermination of the evolution of living organisms, to be understood in terms of the genericity of the trajectories and of changes of phase spaces. As a result, similarly as classical dynamics and quantum mechanics propose two causally different notions of randomness (a dice and the spin-up spin-down are random in a very different sens), so our approach to biological phenomena suggests a third form of randomness: the indetermination locates itself at the level of the very space of phases or evolutions and coexists with a relative structural stability of individual biolons.

The extension of critical situations, conjunctly with the intrication of the levels of organization and their effects of reciprocal "resonance", proposes an intelligibility of the physical type, inasmuch as physics succeeds in providing us with adequate metaphors. As quantum physics has succeeded to do with regard to classical mechanics, it would be necessary to provide ourselves with autonomous concepts and, if ever possible, mathematical structures, in order to better grasp the fields of living phenomena and their dynamics ${ }^{9}$.

\section{Another view on stability and variability.}

We have mentioned earlier the stabilizing role of regulation and integration for the intertwining and the intrication of levels of organization. Our analysis, in terms of the genericity of the trajectories and dynamics of phase spaces should contribute to making intelligible the fact that the phenomenal life is characterized by the preservation of a few key invariants not only due to stability, but also to variability. For this reason, we have considered the intertwining and the interactions of organization levels on the one hand as one of the components of stabilization, and on the other as forming part of biological indetermination (as resonances destabilizing each level of organization). Following variability, typically, biolons all differ from one another: the individuals of a species are not and must not be identical. Even cells have a certain form of "individuality". Indeed, variability is at the centre of evolution and, in conjunction with individuation, it is also at the basis of ontogenesis. Clearly, here lies another singularity of living phenomena in reference to the physical descriptions, where individual entities of a "same type" are all identical and their description can be, conceptually as well as mathematically, perfectly stable (the specificity of physical trajectories which we have addressed).

\subsection{Biolons as attractors and individual trajectory.}

\footnotetext{
${ }^{9}$ The theory of viability (see [Aubin, 1991]) proposes a mathematical analysis which is close, but different, to the approach which we propose: we replace the evolution equation $(\mathrm{dx} / \mathrm{dt}=\ldots)$ membership within a set of possible evolutions $(\mathrm{dx} / \mathrm{dt} \in \ldots)$. However, the function $\mathrm{x}(\mathrm{t})$ and the set which determine this passage are given beforehand and contain, according to our interpretation, the list of all possible future spaces, with their geodesics ; $\mathrm{x}(\mathrm{t})$, particularly, represents a "trajectory" of phase spaces, instead of a trajectory within one phase space. It is there, we believe, that we must shift form a determined trajectory to an intrinsic indetermination, comparable to the possible and indeterminate paths of quantum physics, but concerning the phase space itself. Moreover, as we have stressed many times, within each space, it is the generic trajectories that play an important role, not only the geodesics.
} 
Let's now try to understand this approach, described by indeterministic dynamics within globally stable frameworks, and this stability/instability dialectic, in different terms, which are nevertheless compatible with the former.

On the one hand, a species presents a global structural stability, whereas its members may display different variations in their nature and behavior. Likewise, in its own time scale, an individual is (relatively) stable, while its cells evolve and die, each according to a different path. In one or the other case, the global structure is essentially (but not entirely) preserved while the local variations take place. If we take a physical analogy, the greater biolon (a species, a metazoan) can be described by means of the geometry of an attractor, of which the global dynamic is essentially stable as long as it remains within a same phase space. The relative instability of individual trajectories within the attractor, those of the smaller biolons in it (the cells in a metazoan, the individuals in a species), contributes to variability of the global structure. Yet, the local biolons, as part of orgons, are stabilized by the function (of the orgon they may belong to - a population in the case of a species). Thus, the functionality contributes to the local and global stability, by maintaining the function. While their minor instabilities constitute adaptability, by (minor) changes in the individual trajectories (or, say, by variations in the fractal structure of lungs yields changes of survival in changing environments).

We can understand the change of phase spaces as the toppling of the attractor into another space, with the characteristics of indetermination, which we have discussed. The difficulty would then consist in the search for the correct classes of universality, or of the great biological invariants, such as, for instance, absolute numbers (the clocks of living matter, etc.) which concern the species, even entire phyla, and which would allow to speak of a toppling of the "same" attractor.

This dynamic stability is as compatible with the variations and even the instability of the individual trajectories, which may fall within a same attractor (the smaller biolons, included within the largest - a metazoan, a cell, respectively). In this sense, a biolon of the intermediate level (a metazoan) constitutes a trajectory for a species, the latter being considered as an attractor; at the same time, this biolon behaves as a stabilizing attractor for is own component, its cells.

As we have observed, in physics, the dynamic of the trajectory is given by the geodesic within a predefined phase space; thus, it is selected according to the highest stability and generally in a unique way. In other words, it is specific and optimal in the sense given by the meric space, a measure. In contrast, individual biolons are, in our opinion, represented by different and generic simultaneous paths, and simply submitted to compatibility constraints with conditions at the boundaries (which, in turn, do not have to be stable and can be modified by continuous processes). This approach would enable to simultaneously capture the properties associated to:

- the interaction of relative global stability and individual variability, as attractor $v s$. trajectory;

- compatibility instead of "optimality" of geodesics, as recalled above, given that trajectories must only remain within the evolutionary boundaries of the attractor.

Each individual trajectory would thus find its origin in an instable situation and would actualize various contextual potentialities (typically the first mitosis in the embryogenesis, 
within the same genotype) and would evolve according to the potentialities accessible within the framework of the global structural stability.

Natural selection may reduce variability at the species level, all the while increasing it at the individual level. The more or less tolerable pathologies would rather concern the cells within an individual. Naturally, this conceptual mode perfectly adapts to the above-proposed notion of extended critical situation, as paradigmatic state of biolons. Besides, it may also be seen as a generalization of Waddington's notion of chreode ([Waddington, 1977]), by the aspects of genericity and indetermination upon which we have insisted.

Notice that this schema refers solely to biolons, at various levels, given that only biolons are concerned by identities (identities preserved by changes). Orgons would rather constitute, through the energetic exchanges and the functional activities to which they are the locus, the material support to stability and to variation: an orgon is not a trajectory ${ }^{10}$, but may be at the origin of the continuous variation of the individual represented by the trajectory.

Physical paradigms have aided us in formulating these notions, which are not of physical nature proper. We would like to remind, once more, that all the while working within a monist framework, we face various phenomenalities, such at least as they are co-constituted with our living and historical being. Over the course of history, these phenomenalities have been made (partially) intelligible, by their organization using various conceptual structures (and if possible, mathematical ones) - especially over the course of the $\mathrm{XX}^{\text {th }}$ century, so rich in science, before aiming toward "unification". A synthesis, in fact, is far from being obvious; it must be constructed by means of a new (and mathematical) conceptual unification as its long term objective. As it has occurred in physics, the conceptual and technical unification with the biological sciences will be possible only after having specified, we believe, the causal structure and the dynamics specific to each phenomenal level, in its theoretical and experimental autonomy and after having established the conceptual bridges connecting one intelligibility to another, without necessarily, or immediately, reducing one to the other.

\footnotetext{
${ }^{10}$ Let's remind that a faltering orgon may be replaced by a "prosthesis", an artifact, likely to serve its purpose without decisively affecting the biolon to which it participates (organs within an organism, for instance). This is generally not possible for biolons: in no way we can replace an individual cell.
} 


\section{References}

(Preliminary or revised versions of Longo's papers are downloadable from

http://www.di.ens.fr/users/longo ).

Aubin, J.P. Viability theory, 1991, Birkhauser.

Bailly F., "L'anneau des disciplines”, Rev. Int. Syst., 5, n³, 1991.

Bailly F., Gaill F., Mosseri R., "Orgons and Biolons" in Theoretical Biology: phenomenological analysis and quantum analogies, Acta Biotheor., 41, p.3, 17, 1993.

Bailly F., Longo G. "Objective and Epistemic Complexity in Biology". Invited lecture, Proceedings of the International Conference on Theoretical Neurobiology, (N. D. Singh, ed.), National Brain Research Centre, New Delhi, INDIA, pp. 62 - 79, 2003.

Bailly F., Longo G. "Space, time and cognition. From The Standpoint of Mathematics and Natural Science". In Causality and Mind, (Peruzzi ed.), Benjamins, Amsterdam, pp. 149-199, 2004.

Bailly F., Longo G. Mathématiques et sciences de la nature. La singularité physique du vivant. Hermann, Paris, 2006.

Bailly F., Longo G. "Geometric schemes for biological time". In preparation, draft downloadable (version française dans Logique du vivant (J. Boniface, P.A. Miquel, eds), n. 13, Noesis-Vrin à paraître, 2008).

Bak P., C. Tang C. and Wiesenfeld K. "Self-organized criticality". Physical Review A 38: 364--374, 1988.

Barenblatt, G.I. Scaling, self-similarity and intermediate asymptotics. Cambridge texts in applied mathematics, n. 14, CUP, 1996.

Bernard-Weil E., Stratégies paradoxales en bio-médecine et sciences humaines, Paris, L'Harmattan 2002.

Binney J., Dowrick N.J., Fisher A.J., Newman M.E.J.. The Theory of Critical Phenomena: An Introduction to the Renormalization Group. Oxford U. P., 1992.

Bourgine P., Stewart J. “Autopoïesis and Cognition”, Artificial Life, 10,3, 2004.

Delamotte B., "A hint of renormalization", Am. J. Phys. 72, 170, 2004.

Edelman G., Tononi G. A Universe of Consciousness. How Matter Becomes Immagination, Basic Books, 2000.

Goodwin B.C., Kauffman S.A., Murray J.D., "Is Morphogenesis inherently robust?", J. Theoret. Bio., 1993.

Gould S.J., L'éventail du vivant, Paris, Seuil, 1997.

Horsfield K., "Morphology of branching trees related to entropy", Respir. Physiol., 29, p.179, 1977.

Jensen H. J., Self-Organized Criticality, Emergent Complex Behavior in Physical and Biological Systems. Cambridge lectures in Physics, 1998.

Kauffman S.A., Origins of Order: Self Organization and Selection in Evolution, Oxford U. P., 1993.

Kauffman S., At home in the Universe, Oxford UP, 1995.

Jean R. V. Phyllotaxis : a systemic study in plant morphogenesis, Cambridge University Press, 1994

Lecointre G., Le Guyader H., Classification phylogénétique du vivant, Paris, Belin 2001

Lefèvre J., "Teleonomical optimization of a fractal model of the pulmonary arterial bed", Journ. Theor. Biol., 102, p.225, 1983.

Longo G. "Laplace, Turing and the "imitation game" impossible geometry: randomness, determinism and programs in Turing's test". In Epstein, R., Roberts, G. \& Beber, G. (Eds.). The Turing Test Sourcebook. Dordrecht, The Netherlands: Kluwer, 2007.

Mandelbrot B., The fractal geometry of nature, New York, W.H. Freeman, 1982.

Maturana H., Mpdozis J., "El origen de las especies por medio de la deriva natural" rvista Chilena de Historia Natural, 73, 261-310, 2000.

Nicolis G., "Dissipative systems", Rev. Prog. Phys., IL, p. 873, 1986.

Nicolis G., Prigogine I, A la rencontre du complexe, PUF, 1992. 
Nicolis G., Prigogine I., Self-organization in non-equilibrium systems, Wiley, New York, 1977.

Rosen R., Life itself: A comprehensive Enquire into the Nature, Origin and Fabrication of Life, New York, Columbia University Press, 1991.

Rossi E., Lloyd D., Ultradian Rhythms in Life Processes: Inquiry into Fundamental Principles of Chronobiology and Psychobiology. Springer-Verlag Berlin and Heidelberg, 1992.

Solé R., Goodwin B., Signs of Life, Basic Books, 2000.

Soto A., Sonnenschein C., "The somatic mutation theory of cancer : growing problems with this paradigm ?" BioEssays, $26: 1097-1107,2004$.

Stewart J. "La modélisation en biologie", in Enquête sur le concept de modèle, (Nouvel P., ed.), Presses Universitaire de France, 2002.

Varela F. J.; Maturana, H. R.; \& Uribe, R. "Autopoiesis: the organization of living systems, its characterization and a model". Biosystems 5 187-196, 1974.

Varela F., Autonomie et connaissance, Seuil, Paris, 1989.

Varela F. "The Specious Present: A Neurophenomenology of Time Consciousness", in : Naturalizing Phenomenology (J. Petitot, F. Varela, B. Pachoud, J-M. Roy, Eds.) Stanford University Press 1999.

Waddington C.H., "Stabilization of Systems, Chreods and Epigenetic Landscapes", Futures, 9(2), p.139, , 18, 1977.

West G., Brown J., Enquist B., "A general model for the origin of allometric scaling laws in biology", Science, CCLXXVI, p.122, 1997. 